\title{
Anti-periodic solutions for nonlinear evolution equations
}

\author{
Yi Cheng ${ }^{1,2}$, Fuzhong Cong ${ }^{1 *}$ and Hongtu Hua ${ }^{1,2}$
}

\author{
"Correspondence: \\ congfz67@126.com \\ Fundamental Department, \\ Aviation University of Air Force, \\ Changchun, 130022, People's \\ Republic of China \\ Full list of author information is \\ available at the end of the article
}

\begin{abstract}
In this paper, we use the homotopy method to establish the existence and uniqueness of anti-periodic solutions for the nonlinear anti-periodic problem

$$
\left\{\begin{array}{l}
\dot{x}+A(t, x)+B x=f(t) \quad \text { a.e. } t \in R \\
x(t+T)=-x(t)
\end{array}\right.
$$

where $A(t, x)$ is a nonlinear map and $B$ is a bounded linear operator from $R^{N}$ to $R^{N}$. Sufficient conditions for the existence of the solution set are presented. Also, we consider the nonlinear evolution problems with a perturbation term which is multivalued. We show that, for this problem, the solution set is nonempty and weakly compact in $W^{1,2}\left(I, R^{N}\right)$ for the case of convex valued perturbation and prove the existence theorems of anti-periodic solutions for the nonconvex case. All illustrative examples are provided.
\end{abstract}

Keywords: anti-periodic solution; evolution equation; Leray-Schauder alternative theorem; measurable selection; continuous selection

\section{Introduction}

Anti-periodic problems have important applications in auto-control, partial differential equations and engineering, and they have been studied extensively in the past ten years. For example, anti-periodic trigonometric polynomials are important in the study of interpolation problems [1], and anti-periodic wavelets are discussed in [2]. Recently, antiperiodic boundary conditions have been considered for the Schrödinger and Hill differential operator [3, 4]. Also, anti-periodic boundary conditions appear in the study of difference equations $[5,6]$. Moreover, anti-periodic boundary conditions appear in physics in a variety of situations, see [7-10].

The study of anti-periodic solutions for nonlinear evolution equations was initiated by Okochi [11]. Since then, many authors have been devoted to investigation of the existence of anti-periodic solutions to nonlinear evolution equations in Hilbert spaces. For the details, see [12-17] and the references therein. In [15], Chen studied the anti-periodic solution for the following first-order semilinear evolution equation:

$$
\left\{\begin{array}{l}
\dot{u}+A u(t)+f(t, u)=0 \quad \text { a.e. } t \in R \\
u(t+T)=-u(t)
\end{array}\right.
$$


where $A: R^{N} \rightarrow R^{N}$ is a matrix, $f: R \times R^{N} \rightarrow R^{N}$ is a continuous function satisfying $f(t+$ $T, u)=-f(t,-u)$ for all $(t, u) \in R \times R^{N}$. Here they assume that $f(t, u)$ is a uniform bound with respect to $u$ and $\frac{T^{2}}{4}\left\|A^{2}\right\|<1$. We do not need these assumptions and consider the following semilinear anti-periodic problem:

$$
\left\{\begin{array}{l}
\dot{x}+A(t, x)+B x=f(t) \quad \text { a.e. } t \in R \\
x(t+T)=-x(t)
\end{array}\right.
$$

where $A: R^{N} \rightarrow R^{N}$ is a hemicontinuous function satisfying $A(t+T, x)=-A(t,-x), f: R \rightarrow$ $R^{N}$ is a measurable function satisfying $f(t+T)=-f(t)$ for all $t \in R$ and $B$ is a bounded linear operator from $R^{N}$ to $R^{N}$. We will establish some sufficient conditions for the existence and uniqueness of anti-periodic solutions of Eq. (1.2) by the theory of topological degree.

In addition, we also consider the following nonlinear evolution inclusion problem:

$$
\left\{\begin{array}{l}
\dot{x}+A(t, x)+B x \in F(t, x) \quad \text { a.e. } t \in I, \\
x(T)=-x(0),
\end{array}\right.
$$

where $I=[0, T]$. We refer the reader to the work of $[18,19]$. These works focused on the problem in which the multivalued term $F(t, x)$ is an even lower semi-continuous convex function with a compact assumption. But, in this paper, we prove the existence theorems of anti-periodic solutions for the cases of a convex and of a nonconvex valued perturbation term which is multivalued based on the techniques and results of the theory of set-valued analysis and the Leray-Schauder fixed point theorem. As far as we know, there are few papers which deal with this type of anti-periodic problems. For recent developments involving the existence of anti-periodic solutions of differential equations, inequalities and other interesting results on anti-periodic boundary value problems, the reader is referred to $[20-27]$ and the references therein.

On the one hand, it is well known that the neural networks have been successfully applied to signal and image processing, pattern recognition and optimization. However, many neural networks with discontinuous neuron activation functions appear in the theoretical study on dynamics of neural networks, see [28, 29]. In order to solve some practical engineering problems, people also need to present new neural networks with discontinuous activation functions. Therefore, developing a new class of neural networks with discontinuous neuron activation functions and giving the conditions of the stability are very valuable in both theory and practice. Motivated by the above discussions, in this paper, we present a class of neural networks with discontinuous neuron activation functions. Based on our results, the existence and uniqueness of the equilibrium point is investigated.

On the other hand, it has been well recognized that differential inclusions, which are certainly of their own interest, provide a useful generalization of control systems governed by differential/evolution equations with control parameters

$$
\dot{x}=f(t, x, u), \quad u \in U(t, x),
$$

where the control sets $U(\cdot, \cdot)$ may also depend on the state variable $x$. Let $F(t, x)=$ $f(t, x, U(t, x))$. Then Eq. (1.4) is reduced to $\dot{x} \in F(t, x)$, which is a particular case of the 
inclusion relation in Eq. (1.3). Hence, we present an example of a nonlinear anti-periodic distributed parameter control system with $a$ priori feedback for our results.

This paper is organized as follows. In Section 2, we state some basic knowledge from multivalued analysis. In Section 3, we first establish the existence of anti-periodic solutions for an evolution equation by the theory of topological degree, and then, by applying the Leray-Schauder fixed point theorem, we prove the existence of anti-periodic solutions for convex and nonconvex cases. Finally, two examples for our results are presented in Section 4.

\section{Preliminaries}

For convenience, we introduce some notations as follows. In Euclidean space, (., .) expresses an inner product, while $|\cdot|$ expresses the Euclidean norm. Let $L^{2}\left([0, T] ; R^{N}\right)$ denote the set of the map $x:[0, T] \rightarrow R^{N}$ which satisfies $\int_{0}^{T}|x|^{2} d t<\infty$, and the norm in $L^{2}\left([0, T] ; R^{N}\right)$ is denoted by $\|x\|_{2}=\left(\int_{0}^{T}|x|^{2} d t\right)^{\frac{1}{2}}$. We recall some basic definitions and facts from multivalued analysis which we shall need in what follows. For details, we refer to the book of Hu and Papageorgiou [30]. Let $I=[0, T],(I, \Sigma)$ be the Lebesgue measurable space and $X$ be a separable Banach space. Denote

$$
P_{(w) k c}(X)=\{A \subset X: \text { nonempty, (weakly) compact and convex }\} .
$$

Let $A \subset P_{f}(X), x \in X$, then the distance form $x$ to $A$ is given by $d(x, A)=\inf \{|x-a|$ : $a \in A$ \}. A multifunction $F: I \rightarrow P_{f}(X)$ is said to be measurable if and only if, for every $z \in X$, the function $t \rightarrow d(z, F(t))=\inf \{\|z-x\|: x \in F(t)\}$ is measurable. A multifunction $G: I \rightarrow 2^{X} \backslash\{\emptyset\}$ is said to be graph measurable if $\operatorname{Gr} G=\{(t, x): x \in G(t)\} \in \Sigma \times B(X)$ with $B(X)$ being the Borel $\sigma$-field of $X$. On $P_{f}(X)$ we can define a generalized metric known in the literature as the 'Hausdorff metric', by setting

$$
h(A, B)=\max \left\{\sup _{a \in A} d(a, B), \sup _{b \in B} d(b, A)\right\}
$$

for all $A, B \in P_{f}(X)$. It is well known that $\left(P_{f}(X), h\right)$ is a complete metric space and $P_{f c}(X)$ is a closed subset of it. When $Z$ is a Hausdorff topological space, a multifunction $G: Z \rightarrow$ $P_{f}(X)$ is said to be $h$-continuous if it is continuous as a function from $Z$ into $\left(P_{f}(X), h\right)$.

Let $Y, Z$ be Hausdorff topological spaces and $G: Y \rightarrow 2^{Z} \backslash\{\phi\}$. We say that $G(\cdot)$ is 'upper semicontinuous (USC)' (resp. 'lower semicontinuous (LSC)'), if for all $C \subseteq Z$ nonempty closed, $G^{-}(C)=\{y \in Y: G(y) \cap C \neq \phi\}$ (resp. $G^{+}(C)=\{y \in Y: G(y) \subseteq C\}$ ) is closed in $Y$. A USC multifunction has a closed graph in $Y \times Z$, while the converse is true if $G$ is locally compact (i.e., for every $y \in Y$, there exists a neighborhood $U$ of $y$ such that $\overline{F(U)}$ is compact in $Z$ ). A multifunction which is both USC and LSC is said to be 'continuous'. If $Y, Z$ are both metric spaces, then the above definition of LSC is equivalent to saying that for all $z \in Z$, $y \rightarrow d_{Z}(z, G(y))=\inf \left\{d_{Z}(z, v): v \in G(y)\right\}$ is upper semicontinuity as $R_{+}$-valued function. Also, lower semicontinuity is equivalent to saying that if $y_{n} \rightarrow y$ in $Y$ as $n \rightarrow \infty$, then

$$
\begin{aligned}
G(y) & \subseteq \underline{\lim } G\left(y_{n}\right)=\left\{z \in Z: \lim d_{Z}\left(z, G\left(y_{n}\right)\right)=0\right\} \\
& =\left\{z \in Z: z=\lim z_{n}, z_{n} \in G\left(y_{n}\right), n \geq 1\right\} .
\end{aligned}
$$


A set $D \subseteq L^{2}(I, X)$ is said to be 'decomposable', if for every $g_{1}, g_{2} \in D$ and for every $J \subseteq I$ measurable, we have $\chi_{J} g_{1}+\chi_{J} g_{2} \in D$. The following lemmas are still needed in the proof of our main theorems.

Lemma 2.1 (see [31]) If $X$ is a Banach space, $C \subset X$ is nonempty, closed and convex with $0 \in C$, and $G: C \rightarrow P_{k c}(C)$ is an upper semicontinuous multifunction which maps bounded sets into relatively compact sets, then one of the following statements is true:

(i) the set $\Gamma=\{x \in C: x \in \lambda G(x), \lambda \in(0,1)\}$ is unbounded;

(ii) the $G(\cdot)$ has a fixed point, i.e., there exists $x \in C$ such that $x \in G(x)$.

Let $X$ be a Banach space and let $L^{2}(I, X)$ be the Banach space of all functions $u: I \rightarrow X$ which are Bochner integrable. $D\left(L^{2}(I, X)\right)$ denotes the collection of nonempty decomposable subsets of $L^{2}(I, X)$. Now, let us state the Bressan-Colombo continuous selection theorem.

Lemma 2.2 (see [32]) Let $X$ be a separable metric space and let $F: X \rightarrow D\left(L^{2}(I, X)\right)$ be a lower semicontinuous multifunction with closed decomposable values. Then $F$ has a continuous selection.

\section{Main results}

\subsection{The evolution equation}

In this section, let

$$
\begin{aligned}
& C_{T}=\left\{x \mid R \mapsto R^{N} \text { is continuous, } x(t+T)=-x(t)\right\}, \\
& W^{1,2}=\left\{x \in C_{T}: \int_{0}^{T}\left(|x|^{2}+|\dot{x}(t)|^{2}\right) d t<\infty\right\},
\end{aligned}
$$

where $\dot{x}$ is the weak derivative of $x . C_{T}$ is a Banach space under the norm $\|x\|_{c}=\max _{t \in R}|x|$. Equipped with the norm

$$
\|x\|_{1,2}=\left(\int_{0}^{T}\left(|x|^{2}+|\dot{x}(t)|^{2}\right) d t\right)^{\frac{1}{2}},
$$

$W^{1,2}$ becomes a separable Banach space. The following is our main result of this part.

Theorem 3.1 Assume the following hold:

(i) $f(t+T)=-f(t)$ and $A(t+T,-x)=-A(t, x)$ for all $(t, x) \in R \times R^{N}$;

(ii) $t \rightarrow A(t, x)$ is measurable and $f \in L^{2}\left([0, T] ; R^{N}\right)$;

(iii) for each $t \in R$, the operator $A(t, \cdot): R^{N} \rightarrow R^{N}$ is uniformly monotone and hemicontinuous, that is, there exists a constant $p>0$ such that $\left(A\left(t, x_{1}\right)-A\left(t, x_{2}\right), x_{1}-x_{2}\right) \geq p\left|x_{1}-x_{2}\right|^{2}$ for all $x_{1}, x_{2} \in R^{N}$, and the map $s \rightarrow(A(t, x+s z), y)$ is continuous on $[0,1]$ for all $x, y, z \in R^{N}$;

(iv) $B: R^{N} \rightarrow R^{N}$ is a bounded linear operator and there exists $c \in R^{+}$such that

$$
(B x, x) \geq c|x|^{2}, \quad \forall x \in R^{N}
$$

then the problem (1.2) has a unique T-anti-periodic solution. 
In order to complete the proof of Theorem 3.1, we need the following lemmas.

Lemma 3.1 (see [33]) Suppose $\Gamma$ is a bounded open set of a normal space $X$, $f$ is compact in $\bar{\Gamma}$ and $p \in X \backslash f(\partial \Gamma)$. Then the equation $f(x)=p$ has at least one solution in $\Gamma$, provided with $\operatorname{deg}(f, \Gamma, p) \neq 0$.

\section{Lemma 3.2 Consider the equation}

$$
\dot{x}+B x=f(t) \text { a.e. } t \in R,
$$

where $B: R^{N} \rightarrow R^{N}$ is a bounded linear operator, and there exists $c \in R^{+}$such that $(B x, x) \geq$ $c|x|^{2}$ for all $x \in R^{N}, f(t+T)=-f(t)$ and $f \in L^{2}\left([0, T] ; R^{N}\right)$. Then the problem (3.1) has a unique T-anti-periodic solution.

Proof Let $x$ be a solution of (3.1) satisfying the boundary value condition $x(0)=-x(T)$. Then $x$ is a $T$-anti-periodic solution of (3.1). Denote $L x=\dot{x}+B x$ for all $x \in W^{1,2}$, then $L: W^{1,2} \rightarrow L^{2}\left([0, T] ; R^{N}\right)$ is a linear operator.

Firstly, we show $L: W^{1,2} \rightarrow L^{2}\left([0, T] ; R^{N}\right)$ is one to one. Suppose $L\left(x_{1}\right)=L\left(x_{2}\right)$, then $\dot{x}_{1}+$ $B x_{1}=\dot{x}_{2}+B x_{2}$ a.e. $t \in R$, and so $\dot{x}_{1}-\dot{x}_{2}+B x_{1}-B x_{2}=0$ a.e. $t \in R$. Take an inner product above with $x_{1}-x_{2}$ and note that

$$
\left(\dot{x}_{1}-\dot{x}_{2}, x_{1}-x_{2}\right)+\left(B x_{1}-B x_{2}, x_{1}-x_{2}\right)=0 .
$$

By using integration from 0 to $T$ and the relation $x(0)=-x(T)$, one can see that

$$
\int_{0}^{T}\left(\dot{x}_{1}-\dot{x}_{2}, x_{1}-x_{2}\right) d t=0
$$

Since $B$ is a linear bounded operator, then

$$
\left(B x_{1}-B x_{2}, x_{1}-x_{2}\right) \geq c\left|x_{1}-x_{2}\right|^{2}
$$

for some constant $c>0$. It follows that

$$
\begin{aligned}
0 & =\int_{0}^{T}\left(B x_{1}-B x_{2}, x_{1}-x_{2}\right) d t \\
& \geq \int_{0}^{T} c\left|x_{1}-x_{2}\right|^{2} d t \\
& =c\left\|x_{1}-x_{2}\right\|_{2}^{2} \\
& \geq 0 .
\end{aligned}
$$

Hence, $x_{1}=x_{2}$ a.e. $t \in R$.

Next, we claim that $L: W^{1,2} \rightarrow L^{2}\left([0, T] ; R^{N}\right)$ is surjective. For this purpose, consider the Cauchy problem

$$
\left\{\begin{array}{l}
\dot{x}+B x=f(t), \\
x(0)=\eta .
\end{array}\right.
$$


It is well known that the above problem has a unique solution which can be written as follows:

$$
x(t)=e^{B t} \eta+\int_{0}^{t} e^{B(t-s)} f(s) d s
$$

Since $-x(0)=x(T)$, then we have that

$$
-\eta=e^{B T} \eta+\int_{0}^{T} e^{B(T-s)} f(s) d s
$$

By hypothesis (iv), one has that $\left(-I-e^{B T}\right)^{-1}$ exists; therefore, when we take

$$
\eta=\left(-I-e^{B T}\right)^{-1} \times \int_{0}^{t} e^{B(t-s)} f(s) d s
$$

the solution of the problem (3.2) is an anti-periodic solution of the problem (3.1). This completes the proof.

Proof of Theorem 3.1 Consider the homotopic systems of (1.2),

$$
\dot{x}+B x=\lambda f(t)-\lambda A(t, x),
$$

where $\lambda \in[0,1]$. Obviously, $\lambda f(t)-\lambda A(t, x)$ is hemicontinuous.

First, a priori bound of the solution set is derived. We claim that there is a priori bound in $W^{1,2}$ for the possible solution $x(t)$ of (3.3). Take the inner product with $x(t)$, and then integrate from 0 to $T$. It follows that

$$
\int_{0}^{T}(\dot{x}, x) d t+\int_{0}^{T}(B x, x) d t=\lambda \int_{0}^{T}(f, x) d t-\lambda \int_{0}^{T}(A(t, x), x) d t .
$$

Without loss of generality, we assume $A(t, 0)=0$. Since $\int_{0}^{T}(\dot{x}, x) d t=0$, and then

$$
\int_{0}^{T}(B x, x) d t=\lambda \int_{0}^{T}(f, x) d t-\lambda \int_{0}^{T}(A(t, x), x) d t .
$$

By hypothesis (iii), we deduce that

$$
c\|x\|_{2}^{2} \leq \lambda\|f\|_{2}\|x\|_{2},
$$

which implies

$$
\|x\|_{2} \leq M_{1}
$$

for some constant $M_{1}>0$. Hence, there is a constant $\tau \in[0, T]$ such that

$$
|x(\tau)|^{2} \leq M_{2}
$$


for some constant $M_{2}>0$. By (3.3), one has that

$$
\begin{aligned}
(\dot{x}, x) & =\lambda(f, x)-\lambda(A(t, x), x)-(B x, x) \\
& \leq \lambda|f| \cdot|x|-\lambda p|x|^{2} .
\end{aligned}
$$

Integrating above from $\tau$ to $t$, we have that

$$
\left|\int_{\tau}^{t}(\dot{x}, x) d t\right| \leq \int_{0}^{T}|f| \cdot|x| d t \leq\|f\|_{2}\|x\|_{2} .
$$

From $\int_{\tau}^{t}(\dot{x}, x) d t=|x(t)|^{2}-|x(\tau)|^{2}$, we know

$$
\left.|| x(t)\right|^{2}-|x(\tau)|^{2} \mid \leq\|f\|_{2}\|x\|_{2} .
$$

By (3.4) and (3.5), we obtain that there is some constant $M_{3}>0$ (independent of $\lambda$ ) such that

$$
|x(t)| \leq\left(|x(\tau)|^{2}+\|f\|_{2}\|x\|_{2}\right)^{\frac{1}{2}} \leq M_{3}
$$

for any $t \in R$. Thus,

$$
\|x\|_{c}=\max _{t \in R}|x(t)| \leq M_{3} .
$$

Since the operator $A$ is hemicontinuous, and $B$ is a bounded linear operator, we show that

$$
\begin{aligned}
& \|\dot{x}\|_{2} \leq M_{4}, \\
& \|x\|_{1,2} \leq M_{5},
\end{aligned}
$$

where the constants $M_{4}, M_{5}>0$. The claim is proved.

Secondly, we can prove the existence of anti-periodic solutions for Eq. (1.2). Set

$$
\Gamma=\left\{x \in W^{1,2}:\|x\|_{1,2}<M_{5}+1\right\} .
$$

Then $\Gamma$ is a bounded open set in $W^{1,2}$. By Lemma 3.2, it is easy to see that

$$
L^{-1}: L^{2}\left([0, T] ; R^{N}\right) \rightarrow W^{1,2}
$$

is well defined. We define the operator $N: \Gamma \rightarrow W^{1,2}, N(x)=L^{-1}(f-A(t, x))$. Obviously, $N$ is compact. Hence, the fixed point of $N$ in $\bar{\Gamma}$ is the anti-periodic solutions of Eq. (1.2). Let $h_{\lambda}(x): \bar{\Gamma} \times[0,1] \rightarrow W^{1,2}$

$$
h_{\lambda}(x)=x-\lambda N(x) .
$$

By (3.7), we obtain $\theta \bar{\epsilon} h(\partial \Gamma)$. So for each $\lambda \in[0,1]$, then we have that

$$
\begin{aligned}
\operatorname{deg}\left(h_{\lambda}, \Gamma, \theta\right) & =\operatorname{deg}\left(h_{1}, \Gamma, \theta\right) \\
& =\operatorname{deg}(i d-N, \Gamma, \theta)
\end{aligned}
$$




$$
\begin{aligned}
& =\operatorname{deg}\left(h_{0}, \Gamma, \theta\right) \\
& =\operatorname{deg}(i d, \Gamma, \theta)=1,
\end{aligned}
$$

where $i d$ is the identity. Consequently, $N$ has a fixed point in $\Gamma$ by Lemma 3.1. Namely, Eq. (1.2) has an anti-periodic solution.

Next, we prove the uniqueness. Suppose that $x_{1}, x_{2}$ are two solutions of Eq. (1.2). Then

$$
\dot{x}_{1}+A\left(t, x_{1}\right)+B x_{1}=\dot{x}_{2}+A\left(t, x_{2}\right)+B x_{2} .
$$

So,

$$
\dot{x}_{1}-\dot{x}_{2}+A\left(t, x_{1}\right)-A\left(t, x_{2}\right)+B x_{1}-B x_{2}=0 .
$$

Take an inner product above with $x_{1}-x_{2}$ and note that

$$
\left(\dot{x}_{1}-\dot{x}_{2}, x_{1}-x_{2}\right)+\left(A\left(t, x_{1}\right)-A\left(t, x_{2}\right), x_{1}-x_{2}\right)+\left(B x_{1}-B x_{2}, x_{1}-x_{2}\right)=0 .
$$

By using integration from 0 to $T$ and the relation $x(0)=-x(T)$, one can see that

$$
\begin{aligned}
0 & =\int_{0}^{T}\left(A\left(t, x_{1}\right)-A\left(t, x_{2}\right), x_{1}-x_{2}\right) d t+\int_{0}^{T}\left(B x_{1}-B x_{2}, x_{1}-x_{2}\right) d t \\
& \geq \int_{0}^{T}(c+p)\left|x_{1}-x_{2}\right|^{2} d t \\
& =(c+p)\left\|x_{1}-x_{2}\right\|_{2}^{2} \\
& \geq 0
\end{aligned}
$$

Hence, $x_{1}=x_{2}$ a.e. $t \in R$. This ends the proof.

\subsection{The evolution inclusions}

Let $I=[0, T]$ and $C\left(I ; R^{N}\right)$ be all the continuous functions from $I$ to $R^{N}$ with the max norm. Let $C_{\beta}=\left\{v(\cdot) \in C\left(I ; R^{N}\right): v(0)=-v(T)\right\}$, and $W^{1,2}\left(I, R^{N}\right)=\left\{u(\cdot) \in C_{\beta}: \dot{u}(\cdot) \in L^{2}\left(I ; R^{N}\right)\right\}$. $W^{1,2}\left(I, R^{N}\right)$ is a separable Banach space under the norm $\|\cdot\|_{1,2}$.

Consider the following anti-periodic problem:

$$
\left\{\begin{array}{l}
\dot{x}+A(t, x)+B x \in F(t, x) \quad \text { a.e. } t \in I \\
x(T)=-x(0)
\end{array}\right.
$$

where $A: R^{N} \rightarrow R^{N}$ is a hemicontinuous function, $B$ is a bounded linear operator from $R^{N}$ to $R^{N}$, and $F: R \times R^{N} \rightarrow 2^{R^{N}}$ is a multifunction. By a solution $x$ of the problem (3.8), we mean a function $x \in W^{1,2}\left(I, R^{N}\right)$, and there exists a function $f(t) \in F(t, x(t))$ such that

$$
\langle\dot{x}(t), v\rangle+\langle A(t, x(t)), v\rangle+\langle B x, v\rangle=\langle f(t), v\rangle
$$

for all $v \in R^{N}$ and almost all $t \in I$. 
In this section, we prove two existence theorems under the hypothesis that the multivalued nonlinearity $F$ is convex-valued ('convex existence theorem') or nonconvex-valued ('nonconvex existence theorem'). The precise hypotheses on the data of the problem (3.8) are as follows:

$H(A): \quad A: I \times R^{N} \rightarrow R^{N}$ is a nonlinear function such that

(i) $t \rightarrow A(t, x)$ is measurable;

(ii) for each $t \in I$, the operator $A(t, \cdot): R^{N} \rightarrow R^{N}$ is uniformly monotone and hemicontinuous, that is, there exists a constant $p>0$ such that $\left(A\left(t, x_{1}\right)-A\left(t, x_{2}\right), x_{1}-x_{2}\right) \geq p\left|x_{1}-x_{2}\right|^{2}$ for all $x_{1}, x_{2} \in R^{N}$, and the map $s \mapsto(A(t, x+s z), y)$ is continuous on $[0,1]$ for all $x, y, z \in R^{N}$.

$H(B): \quad B: R^{N} \rightarrow R^{N}$ is a bounded linear operator, and there exists $c \in R^{+}$such that

$$
(B x, x) \geq c|x|^{2}, \quad \forall x \in R^{N} .
$$

$H(F)_{1}: F: R \times R^{N} \rightarrow P_{k}\left(R^{N}\right)$ is a multifunction such that

(i) $(t, x) \rightarrow F(t, x)$ is graph measurable;

(ii) for almost all $t \in I, x \rightarrow F(t, x)$ is LSC;

(iii) there exists an nonnegative function $b(\cdot) \in L_{+}^{2}(I)$ and a constant $c_{1}>0$ such that

$$
|F(t, x)|=\sup \{\|f\|: f \in F(t, x)\} \leq b(t)+c_{1}|x|^{\alpha},
$$

for all $x \in R^{N}, t \in T$, where $\alpha<1$ or $\alpha=1$ with $c_{1}<c(c$ in $H(B))$.

$H(F)_{2}: F: I \times R^{N} \rightarrow P_{k c}\left(R^{N}\right)$ is a multifunction such that

(i) $(t, x) \rightarrow F(t, x)$ is graph measurable;

(ii) for almost all $t \in I, x \rightarrow F(t, x)$ has a closed graph; and $H(F)_{1}($ iii) holds.

Theorem 3.2 If hypotheses $H(A), H(B)$ and $H(F)_{1}$ hold, then the problem (3.8) has a solution $x \in W^{1,2}\left(I, R^{N}\right)$.

Proof Let $L x=\dot{x}+A(t, x)+B x$ for all $x \in W^{1,2}\left(I, R^{N}\right)$. By Theorem 3.1, we have $L$ : $W^{1,2}\left(I, R^{N}\right) \rightarrow L^{2}\left([0, T] ; R^{N}\right)$ is one to one and surjective, and so $L^{-1}: L^{2}\left([0, T] ; R^{N}\right) \rightarrow$ $W^{1,2}\left(I, R^{N}\right)$ is well defined. So, we prove that

$$
L^{-1}: L^{2}\left([0, T] ; R^{N}\right) \rightarrow L^{2}\left([0, T] ; R^{N}\right)\left(W^{1,2}\left(I, R^{N}\right) \subset L^{2}\left([0, T] ; R^{N}\right)\right)
$$

is completely continuous (i.e., it is continuous and maps bounded sets into relatively compact sets). To this end, let $K \subset L^{2}\left([0, T] ; R^{N}\right)$ be bounded. We shall show that $L^{-1}(K)$ is relatively compact in $L^{2}\left([0, T] ; R^{N}\right)$. For this purpose, let $x \in L^{-1}(K)$, then $x=L^{-1}(u)$ with $u \in K$. By (3.7), we have $\|x\|_{2} \leq c\|L x\|_{2}=\|u\|_{2} \leq c|K|=c \sup \left\{\|u\|_{2}: u \in K\right\}<+\infty$ and $\|\dot{x}\|_{2} \leq\|u\|_{2}+\|A(x)\|_{2}+\|B x\|_{2} \leq M$ for some constant $M>0$. From these bounds we infer that $L^{-1}(K)$ is bounded in $W^{1,2}\left(I, R^{N}\right)$. But $W^{1,2}\left(I, R^{N}\right)$ is compactly embedded in $L^{2}\left([0, T] ; R^{N}\right)$. Therefore, $L^{-1}(K)$ is relatively compact in $L^{2}\left([0, T] ; R^{N}\right)$. Also, from the fact that $L^{-1}$ is a compact operator, $L^{-1}: L^{2}\left([0, T] ; R^{N}\right) \rightarrow L^{2}\left([0, T] ; R^{N}\right)$ is continuous.

Next, let $N: L^{2}\left([0, T] ; R^{N}\right) \rightarrow 2^{L^{2}\left([0, T] ; R^{N}\right)}$ be the multivalued Nemitsky operator corresponding to $F$ and $N$ be defined by $N(x)=\left\{v \in L^{2}\left([0, T] ; R^{N}\right): v(t) \in F(t, x(t))\right\}$ a.e. on $I$. 
We claim that $N(\cdot)$ has nonempty, closed, decomposable values and is LSC. The closedness and decomposability of the values of $N(\cdot)$ are easy to check. For the nonemptiness, note that if $x \in L^{2}\left([0, T] ; R^{N}\right)$, by hypothesis $H(F)_{1}(\mathrm{i}),(t, x) \rightarrow F(t, x)$ is graph measurable, so we apply Aumann's selection theorem and obtain a measurable map $v: I \rightarrow R^{N}$ such that $v(t) \in F(t, x(t))$ a.e. on $I$. By hypothesis $H(F)_{1}\left(\right.$ iii), $v \in L^{2}\left([0, T] ; R^{N}\right)$. Thus for every $x \in R^{N}, N(x) \neq \emptyset$. To prove the lower semicontinuity of $N(\cdot)$, we only show that every $u \in L^{2}\left([0, T] ; R^{N}\right), x \rightarrow d(u, N(x))$ is a USC $R_{+}$-valued function. Note that

$$
\begin{aligned}
d(u, N(x)) & =\inf \left\{\|u-v\|_{2}: v \in N(x)\right\} \\
& =\inf \left\{\left(\int_{0}^{T}|u(t)-v(t)|^{2} d t\right)^{\frac{1}{2}}: v \in N(x)\right\} \\
& =\left(\int_{0}^{T} \inf \left\{|u(t)-v(t)|_{2}: v \in F(t, x)\right\} d t\right)^{\frac{1}{2}} \\
& =\left(\int_{0}^{T} d(u(t), F(t, x))^{2} d t\right)^{\frac{1}{2}}
\end{aligned}
$$

(see Hiai and Umegaki [34] Th. 2.2). We shall show that for every $\lambda \geq 0$, the superlevel set $U_{\lambda}=\left\{x \in L^{2}\left([0, T] ; R^{N}\right): d(u, N(x)) \geq \lambda\right\}$ is closed in $L^{2}\left([0, T] ; R^{N}\right)$. Let $\left\{x_{n}\right\}_{n \geq 1} \subseteq U_{\lambda}$ and assume that $x_{n} \rightarrow x$ in $L^{2}\left([0, T] ; R^{N}\right)$. By passing to a subsequence, if necessary, we may assume that $x_{n}(t) \rightarrow x(t)$ a.e. on $I$ as $n \rightarrow \infty$. By hypothesis $H(F)_{1}(\mathrm{ii}), x \rightarrow d(u, F(t, x))$ is an upper semicontinuous $R_{+}$-valued function. So, via Fatou's lemma, we have

$$
\begin{aligned}
& \left.\lambda^{2} \leq \varlimsup-\varlimsup i d\left(u, N\left(x_{n}\right)\right)\right]^{2}=\varlimsup \int_{0}^{T}\left[d\left(u(t), F\left(t, x_{n}\right)\right)\right]^{2} d x \\
& \leq \int_{0}^{T} \varlimsup \lim \left[d\left(u(t), F\left(t, x_{n}\right)\right)\right]^{2} d x \\
& \leq \int_{0}^{T}[d(u, F(t, x))]^{2} d t=[d(u, N(x))]^{2} .
\end{aligned}
$$

Therefore, $x \in U_{\lambda}$ and this proves the LSC of $N(\cdot)$.

We apply Lemma 2.2 and obtain a continuous map $f: L^{2}\left([0, T] ; R^{N}\right) \rightarrow L^{2}\left([0, T] ; R^{N}\right)$ such that $f(x) \in N(x)$. To finish our proof, we only need to solve the fixed point problem: $x=L^{-1} f(x)$.

We claim that the set $\Gamma=\left\{x \in L^{2}\left([0, T] ; R^{N}\right): x=\sigma L^{-1} f(x), \sigma \in(0,1)\right\}$ is bounded. Let $x \in \Gamma$, then $x=\sigma L^{-1} f(x)$. By hypothesis $H(F)_{1}($ iii), we can derive

$$
|f(x)| \leq b(t)+c_{1}|x|^{\alpha}
$$

then

$$
\begin{aligned}
\|f(x)\|_{2} & \leq\|b\|_{2}+\left\|c_{1}|x|^{\alpha}\right\|_{2} \\
& \leq\|b\|_{2}+\left(\left\|c_{1}^{2}\right\|_{\frac{1}{1-\alpha}}\left\||x|^{2 \alpha}\right\|_{\frac{1}{\alpha}}\right)^{1 / 2} \\
& =\|b\|_{2}+\left\|c_{1}\right\|_{\frac{2}{1-\alpha}}\|x\|_{2}^{\alpha} \\
& =\|b\|_{2}+c_{1} T^{\frac{2}{1-\alpha}}\|x\|_{2}^{\alpha}
\end{aligned}
$$


with $\alpha<1$. By (3.6), we get that

$$
\|x\|_{2} \leq c_{2}\|f\|_{2}
$$

for some constant $c_{2}>0$. So, we have that

$$
\|x\|_{2} \leq c_{2}\|b\|_{2}+c_{1} c_{2} T^{\frac{2}{1-\alpha}}\|x\|_{2}^{\alpha}
$$

Thus, we can find a constant $c_{3}>0$ such that $\|x\|_{2} \leq c_{3}$. If $\alpha=1$, we can also find a constant $\overline{c_{3}}=\frac{\|b\|_{2}}{c-c_{1}}>0$ such that $\|x\|_{2} \leq \overline{c_{3}}$. Similar to the estimation of (3.7), we have that

$$
\|x\|_{c}=\max _{t \in R}|x(t)| \leq c_{4}
$$

for some constant $c_{4}>0$. So, $\Gamma$ is bounded in $L^{2}\left([0, T] ; R^{N}\right)$. Invoking Leray-Schauder's alternative theorem, we obtain there exists $x \in W^{1,2}\left(I, R^{N}\right)$ such that $x=L^{-1} f(x), x$ is a solution of the problem (3.8). This ends the proof.

Theorem 3.3 If hypotheses $H(A), H(B)$ and $H(F)_{2}$ hold, then the problem (3.8) has a solution $x \in W^{1,2}\left(I, R^{N}\right)$. Moreover, the solution set is weakly compact in $W^{1,2}\left(I, R^{N}\right)$.

Proof The proof is as that of Theorem 3.2. So, we only present those particular points where the two proofs differ.

In this case, the multivalued Nemistsky operator $N: L^{2}\left([0, T] ; R^{N}\right) \rightarrow 2^{L^{2}\left([0, T] ; R^{N}\right)}$ has nonempty closed, convex values in $L^{2}\left([0, T] ; R^{N}\right)$ and is USC. The closedness and convexity of the values of $N(\cdot)$ are clear. To prove the nonemptiness, let $x \in W^{1,2}\left(I, R^{N}\right)$ and $\left\{s_{n}\right\}_{n \geq 1}$ be a sequence of step functions such that

$$
s_{n}(t) \rightarrow x \quad \text { and } \quad\left|s_{n}(t)\right| \leq|x(t)| \text { a.e. on } I .
$$

Then by virtue of hypothesis $H(F)_{2}(\mathrm{i})$, for every $n \geq 1, t \rightarrow F\left(t, s_{n}\right)$ admits a measurable selector $f_{n}(t)$. From hypothesis $H(F)_{2}$ (iii), we have that there exists a constant $c_{5}>0$ such that

$$
\sup _{f_{n} \in F\left(t, s_{n}\right)}\left\|f_{n}\right\|_{2} \leq\|b\|_{2}+c_{5}\|x\|_{2}^{\alpha}
$$

So $\left\{f_{n}\right\}_{n \geq 1}$ is uniformly integrable. By the Dunford-Pettis theorem, and by passing to a subsequence if necessary, we may assume that $f_{n} \rightarrow f$ weakly in $L^{2}\left([0, T] ; R^{N}\right)$. Then from Theorem 3.1 in [35], we have

$$
f(t) \in \overline{\operatorname{conv}} \varlimsup\left\{f_{n}(t)\right\}_{n \geq 1} \subseteq \overline{\operatorname{conv}} \varlimsup F\left(t, s_{n}\right) \subseteq F(t, x) \quad \text { a.e. on } I
$$

the last inclusion being a consequence of hypothesis $H(F)_{2}$ (ii). So $f \in N(x)$. Thus we prove the nonemptiness of $N(\cdot)$.

Next, we show that $N(\cdot)$ is USC from $W^{1,2}\left(I, R^{N}\right)$ into $L^{2}\left([0, T] ; R^{N}\right)_{w}$. Let $C$ be a nonempty and weakly closed subset of $L^{2}\left([0, T] ; R^{N}\right)$. We need to show that the set

$$
N^{-1}(C)=\{x \in D(L): N(x) \cap C \neq \phi\}
$$


is closed. Let $\left\{x_{n}\right\}_{n \geq 1} \subseteq N^{-1}(C)$ and assume $x_{n} \rightarrow x$ in $W^{1,2}\left(I, R^{N}\right)$. Passing to a subsequence, we can get that $x_{n}(t) \rightarrow x(t)$ a.e. on $I$. Let $f_{n} \in N\left(x_{n}\right) \cap C, n \geq 1$. Then by virtue of hypothesis $H(F)_{2}$ (iii) and the Dunford-Pettis theorem, we may assume that $f_{n} \rightarrow f \in C$ weakly in $L^{2}\left([0, T] ; R^{N}\right)$. As before, we have

$$
f(t) \in \overline{\operatorname{conv}} \varlimsup\left\{f_{n}(t)\right\}_{n \geq 1} \subseteq \overline{\operatorname{conv}} \varlimsup F\left(t, x_{n}\right) \subseteq F(t, x) \quad \text { a.e. on } I,
$$

then $f \in N(x) \cap C$, i.e., $N^{-}(C)$ is closed in $W^{1,2}\left(I, R^{N}\right)$. This proves the upper semicontinuity of $N(\cdot)$ from $W^{1,2}\left(I, R^{N}\right)$ into $L^{2}\left([0, T] ; R^{N}\right)_{w}$.

We consider the following fixed point problem:

$$
x \in L^{-1} N(x) .
$$

Recalling that $L^{-1}: L^{2}\left([0, T] ; R^{N}\right) \rightarrow L^{2}\left([0, T] ; R^{N}\right)$ is completely continuous, we see that $L^{-1} N: L^{2}\left([0, T] ; R^{N}\right) \rightarrow P_{k c}\left(L^{2}\left([0, T] ; R^{N}\right)\right)$ is USC and maps bounded sets into relatively compact sets. We easily check that the set

$$
\Gamma_{1}=\left\{u \in L^{2}\left([0, T] ; R^{N}\right): u \in \sigma L^{-1} N(u), \sigma \in(0,1)\right\}
$$

is bounded, as in the proof of Theorem 3.2. Invoking Lemma 2.1, there exists $u \in$ $W^{1,2}\left(I, R^{N}\right)$ such that $u \in L^{-1} N(u)$. Evidently, this is a solution of the problem (3.8).

Let $S$ denote the solution set of the problem (3.8). As in the proof of Theorem 3.2, we have that $|S|=\sup \left\{\|u\|_{1,2}: u \in S\right\} \leq M$, where $M>0$. By virtue of hypothesis $H(F)_{2}$ (iii) and the Dunford-Pettis theorem, we may assume that $u_{n} \rightarrow u$ weakly in $W^{1,2}\left(I, R^{N}\right)$. As before, we have

$$
L u \in \overline{\operatorname{conv}} \varlimsup\left\{L u_{n}\right\}_{n \geq 1} \subseteq \overline{\operatorname{conv}} \varlimsup \overline{\lim } F\left(t, u_{n}\right) \subseteq F(t, u) \text { a.e. on } I,
$$

then $u \in S$, hence $S$ is weakly compact in $W^{1,2}\left(I, R^{N}\right)$.

\section{Examples}

As an application of the previous results, we introduce two examples. Consider a class of neural networks described by the system of differential equations

$$
\dot{x}=-B x(t)+A g(x)+I(t)
$$

where $x=\left(x_{1}, x_{2}, \ldots, x_{N}\right)^{T} \in R^{N}$ is the vector of neuron state, $A=\operatorname{diag}\left(a_{1}, a_{2}, \ldots, a_{N}\right)$ is an $N \times N$ diagonal matrix, where $a_{i}<0, i=1,2, \ldots, N$, are the neuron self-inhibitions; $B=$ $\left(b_{i j}\right)$ is an $N \times N$ positive definite matrix, which represents the neuron interconnection matrix. Moreover, $g(x)=\left(g_{1}\left(x_{1}\right), g_{2}\left(x_{2}\right), \ldots, g_{N}\left(x_{N}\right)\right)^{T}: R^{N} \rightarrow R^{N}$ is a mapping where $g_{i}: i=$ $1,2, \ldots, N$, represents the neuron input-output activation and $I(t)=\left(I_{1}(t), I_{2}(t), \ldots, I_{N}(t)\right)^{T}$ : $R \rightarrow R^{N}$ is the mapping of neuron inputs.

We set $A(t, x)=-A g(x)$. It is easy to check $A(t, x)$ satisfies the condition of Theorem 3.1. Moreover, $I(t)$ is bounded and $B$ is a positive definite matrix. Thus, by Theorem 3.1 we easily obtain the following theorem. 
Theorem 4.1 If for any $x, \bar{x} \in R^{N}$, there exists a constant $\alpha \in R_{+}$such that $(g(x)-g(\bar{x}), x-$ $\bar{x}) \geq \alpha|x-\bar{x}|^{2}$, and $g(-x)=-g(x), I(t+T)=-I(t)$ for all $t \in R, x \in R^{N}$, then the problem (4.1) has a unique anti-periodic solution.

Discontinuous dynamical systems, particularly neural networks with discontinuous activation functions, arise in a number of applications. Further, we need the following assumptions.

$\mathrm{H}(\mathrm{C})$ : We have $I_{i} \in \Omega$, for any $i=1,2, \ldots, N$, where $\Omega$ denotes the class of functions from $R^{N}$ to $R$ which are monotone nondecreasing bounded and have at most a finite number of jump discontinuities in every compact interval.

We note that if $I$ satisfies $H(C)$, then any $I_{i}, i=1,2, \ldots, N$, possesses only isolated jump discontinuities where $I_{i}$ is not necessary defined. Hence for all $x \in R^{N}$, we have

$$
\Phi[I(x)]=\left(\left[I_{1}\left(x_{1}^{-}\right), I_{1}\left(x_{1}^{+}\right)\right],\left[I_{2}\left(x_{2}^{-}\right), I_{2}\left(x_{2}^{+}\right)\right], \ldots,\left[I_{N}\left(x_{N}^{-}\right), I_{N}\left(x_{N}^{+}\right)\right]\right)
$$

where $I_{i}\left(x_{i}^{-}\right)=\underline{\lim }_{\varepsilon \rightarrow x_{i}} I_{i}(\varepsilon), I_{i}\left(x_{i}^{+}\right)=\varlimsup_{\mathcal{\operatorname { l i m }}} x_{i} I_{i}(\varepsilon)$. Thus the differential equations (4.1) become the following differential inclusions:

$$
\dot{x} \in A g(x)-B x(t)+\Phi[I(x)] .
$$

The existence and the stability of the equilibrium point of (4.1) were first discussed in [29] $(I(t)$ is constant). In [28], the authors proved the existence of periodic solutions of (4.1) when $I(t)$ is the continuous periodic input and $g(x)$ is discontinuous.

We set $F(t, x)=\Phi[I(x)]$, it is easy to check $F(t, x)$ satisfies $H(F)_{2}$. Thus, by Theorem 3.3, we obtain the following theorem.

Theorem 4.2 Iffor any $x, \bar{x} \in R^{N}$, there exists a constant $\alpha \in R_{+}$such that $(g(x)-g(\bar{x}), x-$ $\bar{x}) \geq \alpha|x-\bar{x}|^{2}$, and $H(C)$ hold, then the problem (4.1) has a nonempty set of solutions $x \in$ $W^{1,2}\left(I, R^{N}\right)$.

Next, we present an example of a nonlinear anti-periodic distributed parameter control system, with a priori feedback (i.e., state dependent control constraint set). Let $T=[0, b]$, $\dot{x}=\left(\dot{x}_{1}, \dot{x}_{2}, \ldots, \dot{x}_{N}\right)$. We consider the following control system:

$$
\left\{\begin{array}{l}
\dot{x}+a(t, x) x+B x=g(t, x) u(t) \quad \text { a.e. } t \in T, \\
x(0)=-x(b) \\
u(t) \in U(t, x(t)) \quad \text { a.e. } t \in T,
\end{array}\right.
$$

where $B$ is a positive definite matrix. The hypotheses on the data (4.3) are as follows:

$H(a): \quad a: T \times R^{N} \rightarrow R^{+}, g: T \times R^{N} \rightarrow R$ are Carathéodory functions such that, for almost all $t \in T$,

$$
\begin{aligned}
& 0<\theta_{1} \leq a(t, x) \leq \theta_{2}, \\
& |g(t, x)| \leq \eta_{1}(t)+\eta_{2}(t)|x|^{\alpha},
\end{aligned}
$$


with $\theta_{1}, \theta_{2}>0,0<\alpha<1, \eta_{1}(t) \in L_{+}^{2}(T), \eta_{2}(t) \in L^{\infty}(T)$.

$H(U): U: T \times R^{N} \rightarrow P_{k}\left(R^{N}\right)$ is a multifunction such that

(i) for all $x \in R^{N}, t \rightarrow U(t, x)$ is measurable;

(ii) for all $t \in T, x \rightarrow U(t, x)$ is $h$-continuous;

(iii) for almost all $t \in T$ and all $x \in R^{N},|U(t, x)| \leq \gamma$, with $\gamma>0$.

Let $A: T \times R^{N} \rightarrow R^{N}$ be the operator defined by $A(t, x)=a(t, x) x$. Evidently, using hypothesis $H(a)$, it is straightforward to check that $A$ satisfies hypothesis $H(A), B$ satisfies hypothesis $H(B)$. Also, let $F: T \times R^{N} \rightarrow P_{k}\left(R^{N}\right)$ be defined by

$$
F(t, x)=\left\{y \in R^{N}: y(t)=g(t, x(t)) u(t), u(t) \in U(t, x(t)) \text {, a.e. on } T\right\} .
$$

Using hypotheses $H(a)$ and $H(U)$, it is straightforward to check that $F$ satisfies hypothesis $H(F)_{1}$.

Rewrite the problem (4.3) in the following equivalent evolution inclusion form:

$$
\left\{\begin{array}{l}
\dot{x}+A(t, x(t))+B x \in F(t, x) \quad \text { a.e. } t \in T, \\
x(0)=-x(b) .
\end{array}\right.
$$

We can apply Theorem 3.2 on the problem (4.3) and obtain:

Theorem 4.3 If hypotheses $H(a)$ and $H(U)$ hold, then the problem (4.3) has a solution $x \in W^{1,2}\left(I, R^{N}\right)$.

\section{Competing interests}

The authors declare that they have no competing interests.

\section{Authors' contributions}

YC carried out the main part of this manuscript. FC participated in the discussion and corrected the main theorem. $\mathrm{HH}$ provided all examples for our results. All authors read and approved the final manuscript.

\section{Author details}

${ }^{1}$ Fundamental Department, Aviation University of Air Force, Changchun, 130022, People's Republic of China. ${ }^{2}$ Institute of Mathematics, Jilin University, Changchun, 130012, People's Republic of China.

\section{Acknowledgements}

The authors would like to express their sincere appreciation to the reviewer for his/her helpful comments in improving the presentation and quality of the paper. This work is partially supported by NSFC Grants 11171350 and Natural Science Foundation of Jilin Province Grants 201115133. The second author was partially supported by NSFC Grant (11171350) and Natural Science Foundation of Jilin Province (201115133) of China.

Received: 26 April 2012 Accepted: 4 September 2012 Published: 19 September 2012

\section{References}

1. Du, JY, Han, HL, Jin, GX: On trigonometric and paratrigonometric Hermite interpolation. J. Approx. Theory 131, 74-99 (2004)

2. Chen, HL: Antiperiodic wavelets. J. Comput. Math. 14, 32-39 (1996)

3. Djiakov, P, Mityagin, B: Spectral gaps of the periodic Schrödinger operator when its potential is an entire function. Adv. Appl. Math. 31, 562-596 (2003)

4. Djiakov, P, Mityagin, B: Simple and double eigenvalues of the Hill operator with a two-term potential. J. Approx. Theory 135, 70-104 (2005)

5. Cabada, A, Vivero, DR: Existence and uniqueness of solutions of higher-order antiperiodic dynamic equations. Adv. Differ. Equ. 4, 291-310 (2004)

6. Wang, Y, Shi, YM: Eigenvalues of second-order difference equations with periodic and antiperiodic boundary conditions. J. Math. Anal. Appl. 309, 56-69 (2005)

7. Abdurahman, A, Anton, F, Bordes, J: Half-string oscillator approach to string field theory (ghost sector: I). Nucl. Phys. B $397,260-282(1993)$ 
8. Ahn, C, Rim, C: Boundary flows in general coset theories. J. Phys. A 32, 2509-2525 (1999)

9. Kleinert, H, Chervyakov, A: Functional determinants from Wronski Green function. J. Math. Phys. 40, 6044-6051 (1999)

10. Pinsky, S, Tritman, U: Antiperiodic boundary conditions to supersymmetric discrete light cone quantization. Phys. Rev. D 62, $087701(2000)$

11. Okochi, H: On the existence of periodic solutions to nonlinear abstract parabolic equations. J. Math. Soc. Jpn. 40, 541-553 (1988)

12. Aizicovici, S, McKibben, M, Reich, S: Anti-periodic solutions to nonmonotone evolution equations with discontinuous nonlinearities. Nonlinear Anal. 43, 233-251 (2001)

13. Aizicovici, S, Reich, S: Anti-periodic solutions to a class of non-monotone evolution equations. Discrete Contin. Dyn. Syst. 5, 35-42 (1999)

14. Aftabizadeh, AR, Aizicovici, S, Pavel, NH: Anti-periodic boundary value problems for higher order differential equations in Hilbert spaces. Nonlinear Anal. 18, 253-267 (1992)

15. Chen, YQ: Anti-periodic solutions for semilinear evolution equations. J. Math. Anal. Appl. 315, 337-348 (2006)

16. Franco, D, Nieto, JJ, O'Regan, D: Anti-periodic boundary value problem for nonlinear first order ordinary differential equations. Math. Inequal. Appl. 6, 477-485 (2003)

17. Okochi, $\mathrm{H}$ : On the existence of anti-periodic solutions to a nonlinear evolution equation associated with odd subdifferential operators. J. Funct. Anal. 91, 246-258 (1990)

18. Chen, YQ, Cho, YJ, O'Regan, D: Anti-periodic solutions for evolution equations associated with monotone type mappings. Appl. Math. Lett. 23, 1320-1325 (2010)

19. Haraux, A: Anti-periodic solutions of some nonlinear evolution equations. Manuscr. Math. 63, 479-505 (1989)

20. Chen, Y, Kim, J, Li, Y: Viscosity periodic and anti-periodic solutions for nonlinear equations. Nonlinear Funct. Anal. Appl. 10(2), 173-177 (2005)

21. Park, J, Ha, T: Existence of antiperiodic solutions for hemivariational inequalities. Nonlinear Anal. 68(4), 747-767 (2008)

22. Jankowski, T: Ordinary differential equations with nonlinear boundary conditions of antiperiodic type. Comput. Math Appl. 47, 1419-1428 (2004)

23. Franco, D, Nieto, J, O'Regan, D: Anti-periodic boundary value problem for nonlinear first order ordinary differential equations. Math. Inequal. Appl. 6, 477-485 (2003)

24. Chen, YQ, Cho, YJ, O'Regan, D: Anti-periodic solutions for evolution equations associated with monotone type mappings Anti-periodic solutions for evolution equations. Math. Nachr. 278, 356-362 (2005)

25. Liu, Q: Existence of anti-periodic mild solutions for semilinear evolution equations. J. Math. Anal. Appl. 377, 110-120 (2011)

26. Liu, ZH: Anti-periodic solutions to nonlinear evolution equations. J. Funct. Anal. 258, 2026-2033 (2010)

27. Wang, Y: Antiperiodic solutions for dissipative evolution equations. Math. Comput. Model. 51, 715-721 (2010)

28. Wu, HQ: Stability analysis for periodic solution of neural networks with discontinuous neuron activations. Nonlinear Anal., Real World Appl. 10, 1717-1729 (2009)

29. Forti, M, Nistri, P: Global convergence of neural networks with discontinuous neuron activation. IEEE Trans. Circuits Syst. I 50, 1421-1435 (2003)

30. Hu, S, Papageorgiou, NS: Handbook of Multivalued Analysis. Volume I: Theory. Kluwer, Dordrecht (1997)

31. Dugundji, J, Granas, A: Fixed Point Theory. Monogr. Matematyczne, vol. 123, pp. 9-31 (1986)

32. Bressan, A, Colombo, G: Extensions and selection of maps with decomposable values. Stud. Math. 90, 69-86 (1988)

33. Deimling, K: Nonlinear Functional Analysis. Springer, New York (1985)

34. Hiai, F, Umegaki, H: Integrals, conditional expectations and martingales of multivalued functions. J. Multivar. Anal. 7 149-182 (1977)

35. Papageoriou, NS: Convergence theorems for Banach space valued integrable multifunctions. Int. J. Math. Math. Sci. $10,433-442(1987)$

\section{Submit your manuscript to a SpringerOpen ${ }^{\circ}$ journal and benefit from:}

- Convenient online submission

Rigorous peer review

- Immediate publication on acceptance

- Open access: articles freely available online

- High visibility within the field

- Retaining the copyright to your article 